\title{
MPC Based Semi-active Suspension Control for Overtaking Maneuvers
}

\author{
Tamás Hegedűs, Balázs Németh², Péter Gáspár² \\ ${ }^{1}$ Department of Control for Transportation and Vehicle Systems, Faculty of Transportation Engineering and Vehicle Engineering, \\ Budapest University of Technology and Economics, H-1111 Budapest, Stoczek street 2, Hungary \\ 2 Institute for Computer Science and Control (SZTAKI), Eötvös Loránd Research Network (ELKH), H-1111 Budapest, Kende street \\ 13-17, Hungary \\ *Corresponding author, e-mail: hegedus.tamas@mail.bme.hu
}

Received: 18 May 2021, Accepted: 15 June 2021, Published online: 09 August 2021

\begin{abstract}
In this paper, the lateral and vertical control design is presented for autonomous vehicles. The vertical control of the vehicle is based on a semi-active suspension system. In the first step, a decision-making process is made. Based on the results of this algorithm an optimal trajectory is planned. Since the trajectory is known, the lateral accelerations can be computed for the given control horizon. In the second step, the vertical control is achieved, which uses the results of the trajectory planning algorithms. The control design is made by a Model Predictive Control (MPC), in which the sign and the maximum value of the additional force can be taken into account. The main goal in the vertical control process is vehicle roll angle minimization. The results of the algorithm are validated using a high fidelity vehicle dynamics simulation software, CarMaker.
\end{abstract}

Keywords

semi-active suspension, autonomous vehicle, overtaking trajectory

\section{Introduction}

In recent years, technologies related to autonomous vehicles have received much attention. Strict requirements for autonomous vehicles have posed many problems for researchers/companies. A self-driving vehicle must meet a number of criteria, among which safety is the most important. Furthermore, ensuring comfort criteria is also an important step during the control design. Using a vertical dynamics control, comfort level of the passengers can be greatly increased. Active suspension systems can be divided into two main groups: the active and the semi-active systems. The damping coefficient can be regulated using the semi-active suspension within a few milliseconds (Gui et al., 2012).

Several papers are dealing with the control problem related to the semi-active suspensions. There are solutions which use the half-vehicle model during the control process (Kanarachos and Spentzas, 2005). Moreover, using a full vehicle model, more effects can be taken into account during the control design (Fang et al., 2011).

In Fergani et al.'s (2013) work and in Poussot-Vassal et al.'s (2008) work a LPV based solution is presented. Moreover, a Linear Quadratic Regulator (LQR) based solution with an observer is presented in Unger et al.'s (2013) work. A Model Predictive Control (MPC) based approach can be found in Canale et al.'s (2005) work and in Göhrle et al.'s (2013) work. In this paper the goal is to design a semi-active suspension using a MPC. During the control design, the comfort requirements are fulfilled.

In Fig. 1 the structure of the algorithm can be seen. In this paper, the lateral and vertical dynamics of the vehicle are controlled at the same time in order to guarantee safety and comfort requirements. In the first step,

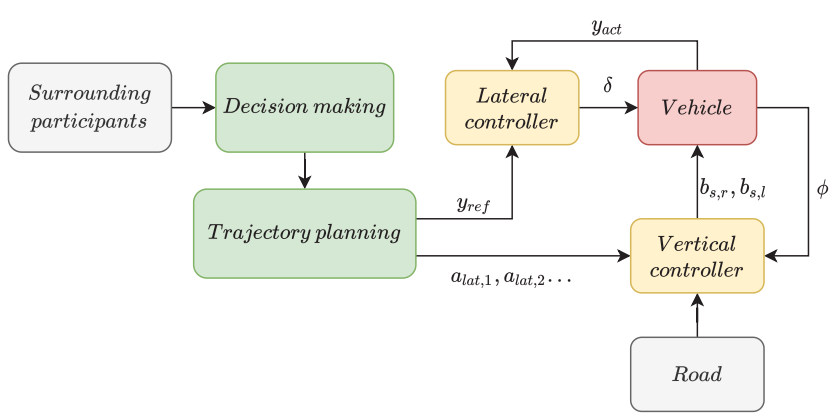

Fig. 1 Structure of the algorithm 
the motion of the surrounding vehicles are predicted. Using this information, the decision making process is made by a graph-based method. The trajectory planning is performed using a model predictive approach. During the trajectory planning, the curvature of the trajectory is minimized, in order to decrease the maximum value of the lateral acceleration. Since the trajectory is known for the controlled vehicle, the lateral accelerations can be computed for the whole trajectory. The values of the lateral accelerations are considered as a disturbance during the vertical control. A Model Predictive Control (MPC) is responsible for the vertical control of the vehicle, in which several limitations are made for the states and for the control input. The lateral controller computes the steering angle $(\delta)$ and the damping coefficients $\left(b_{s, r}, b_{s, f}\right)$ are computed by the vertical MPC.

The structure of the paper is the following: in Section 2 the motion planning is detailed. The trajectory planning algorithm, which uses a model predictive control-based method is introduced in Section 3. The vertical control and the limitations are detailed in Section 4. A comprehensive simulation example can be found in Section 5. Finally, the whole paper is summarized in Section 6.

\section{Motion planning}

In this section, the motion planning of the controlled vehicle is presented. During the trajectory planning, several requirements must be fulfilled at the same time, which can be divided into two main groups. Firstly, the safety requirement must be guaranteed for the controlled vehicle. This requirement can be met by taking into account the limitations of the longitudinal and lateral position of the vehicle. Secondly, comfort requirements play an important role in the trajectory planning process. This means, that the accelerations and the roll angle must also be limited during the maneuver. The accelerations can be limited by the lateral controller, while the roll angle is limited using the vertical controller.

The motion planning algorithm of the vehicle is divided into two main layers. The upper layer is responsible for the decision-making process, which takes into account the surrounding vehicles, while the lower layer is for trajectory planning.

\subsection{Decision making}

The motion prediction of the surrounding vehicles is the basis of the decision-making layer, which is made in the longitudinal and lateral direction at the same time. In this paper, vehicle motions are predicted using density functions, which are determined by a previously recorded dataset. During the determination of the density functions, the Next Generation Simulation (NGSIM) dataset is used, which contains several states of the vehicles along the given road segment. The length of the examined road segment is approximately $640 \mathrm{~m}$ and there can be found 5 lanes.

\subsubsection{Computation of the lateral density functions}

The density function for lateral direction is computed using 300 overtaking trajectories, which are selected from the dataset. Since the measurements can be noisy, the selected dataset cannot be used directly. Using clothoid segments, feasible trajectories are fitted to the measured dataset. In the next step, the overtaking maneuvers are evaluated using the fitted trajectories. In Fig. 2 an example for the measured and the fitted trajectory can be seen. The fitted trajectory is represented with the red line and the raw position information is given by the blue circles.

Based on the fitted trajectories and using the velocity of the vehicle, the value of the lateral acceleration can be determined, with which one overtaking maneuver is

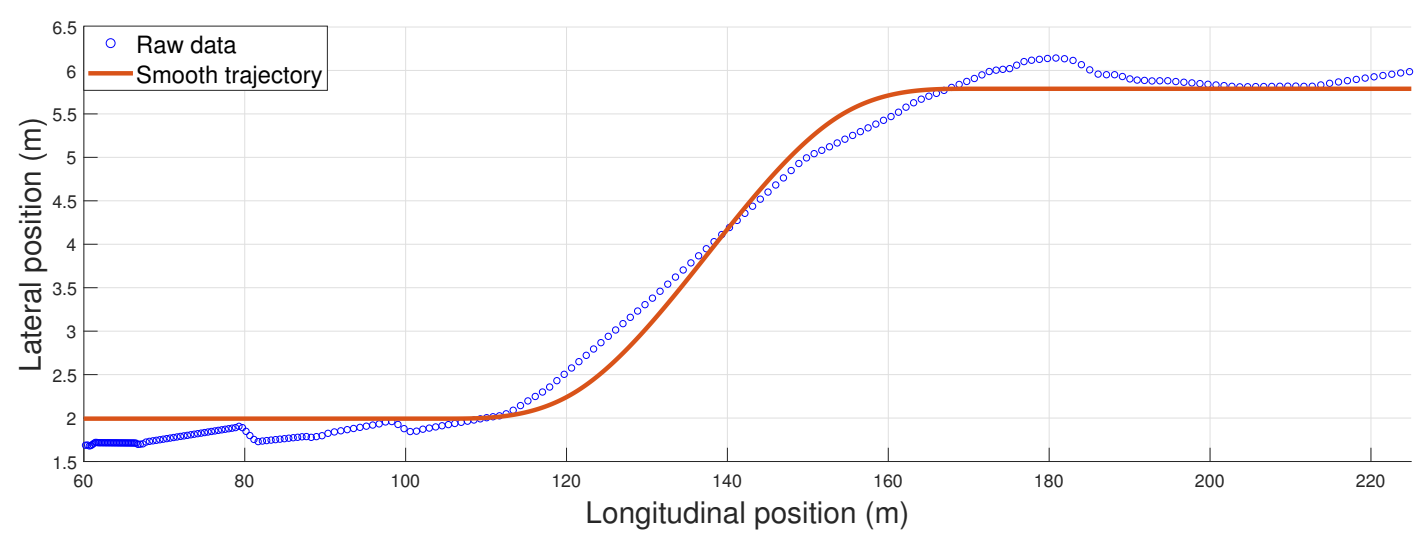

Fig. 2 Fitted overtaking trajectory 
characterized. The calculation of the density function is based on these acceleration values. Gamma distribution function is used to describe the lateral motion of the vehicle [9], which can be formed as Eq. (1):

$$
f_{\text {lat }}(x, \alpha, \beta)=\frac{\beta^{\alpha} x^{\alpha-1} e^{-\beta x}}{\Gamma(\alpha)},
$$

where the parameters $(x, \alpha, \beta)$, are non-negative. Furthermore, the Gamma function is formed as: $\Gamma(\alpha)=\int_{0}^{\infty} x^{\alpha-1} e^{-x} \mathrm{~d} x$.

During motion prediction, the reachability lateral positions for the surrounding vehicles can be calculated using the possible lateral acceleration values.

\subsubsection{Computation of longitudinal density functions}

The determination of the longitudinal motion density functions is also performed using the NGSIM dataset. The main idea is to assume a constant acceleration at a given prediction horizon. Then, based on the dataset, the error between the constant acceleration and the measured values are computed. Using the computed error values, a Gaussian distribution function is fitted, which can be described as Eq. (2):

$f_{\text {long }}\left(a_{\text {long }}, \sigma, \mu\right)=\frac{1}{\sigma \sqrt{2 \pi}} e^{-\frac{1}{2}\left(\frac{a-\mu}{\sigma}\right)^{2}}$,

where $\sigma$ is the standard deviation, $\mu$ gives the mean of the computed dataset and $a_{\text {long }}$ gives the possible longitudinal acceleration of the vehicle. Using this value and the initial velocity value and the possible reachability points can be determined. Moreover, a probability value can also be calculated for the possible longitudinal positions of the given vehicle. In Fig. 3, an example can be seen for the density functions for given time steps.

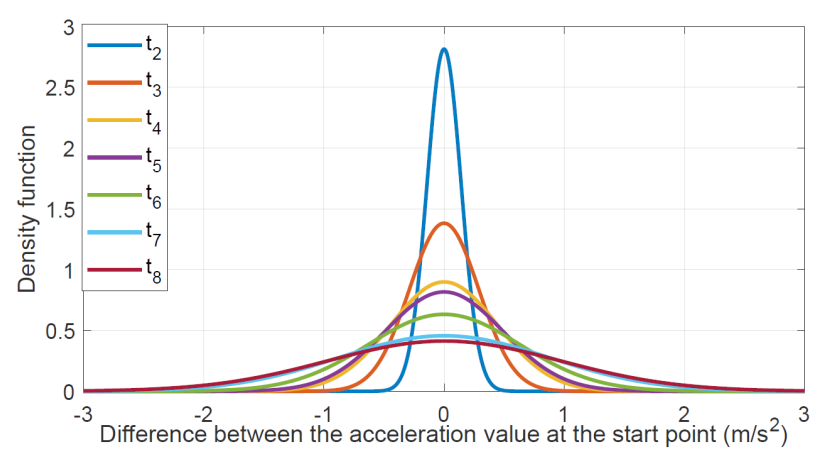

Fig. 3 Density functions for the longitudinal motion

\subsubsection{Motion prediction using density functions}

In the followings, the motion of the surrounding vehicles are predicted using the computed density functions. The motion prediction is essential for the decision making, which is made using a graph-based algorithm. Assuming that the velocities and distances can be measured between the controlled and other vehicles, the density functions are used to predict the future position of the other participants. Using Eqs. (1), (2) the probability can be computed at the given position $\left(x_{1}(t), y_{1}(t)\right)$ as Eq. (3):

$$
P_{\text {lat }}\left(x_{1}, y_{1}\right)=\int_{a_{\text {lat min }}}^{a_{\text {lat, max }}} f_{\text {lat }}(x, \alpha, \beta) \mathrm{d} x \times \int_{0}^{x_{1}} f_{\text {long }}\left(a_{\text {long }}, \sigma, \mu\right) \mathrm{d} a, \text {, (3) }
$$

where $\left(a_{l a t \text {, min }}, a_{l a t, \text { max }}\right)$ gives the maximum and minimum lateral acceleration value, with which the given position can be reached. Furthermore, the whole prediction horizon is divided into discrete points, which can be computed as it is described in (Hegedüs et al., 2020). Using Eq. (3) the probabilities can be computed for each points and the calculated values are assigned to the positions. In the following step, the points are connected with each other, this results in a directed graph (Hegedüs et al., 2020).

$G=(V, \bar{E})$,

where $V$ gives the vertices that are previously calculated. Moreover, $\bar{E}$ gives the edges between the vertices. Two points are connected if the vehicle is able to get from one point to the other, taking into account the physical limits of the vehicle. In Fig. 4 the directed graph can be seen, with which the decision is made for the controlled vehicle.

Using the computed directed graph and a greedy algorithm, a collision-free trajectory can be computed. It is important to note that this trajectory cannot be traced because it is built up using linear segments. However, it

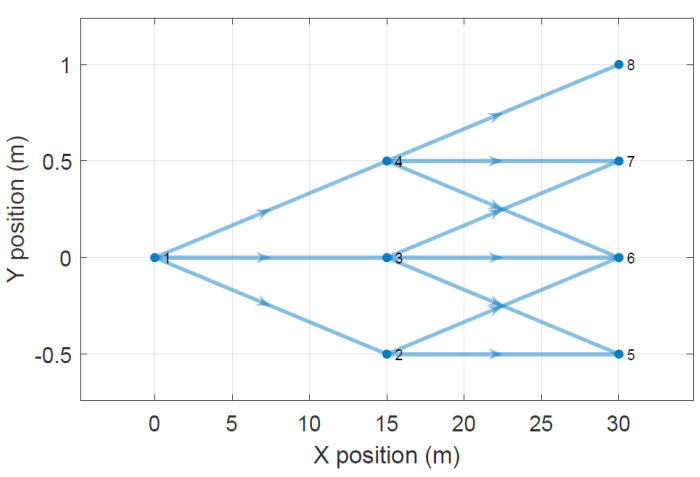

Fig. 4 Graph based decision making 
can be used as a reference trajectory during the feasible trajectory design process. The trajectory is calculated using a model predictive-based method, which is presented in the following subsection.

\section{Trajectory planning of the vehicle}

In this section the trajectory planning is presented. During the trajectory planning, the results of the decision making algorithm are used as a reference vector.

\subsection{Lateral dynamics of the vehicle}

Since the comfort and safety requirements must be satisfied several limitations must be taken into account during the trajectory planning. In the first step, the dynamic vehicle model is presented, which consists of three main equations (Eqs. (5), (6) and (7)):

$$
\begin{aligned}
& I_{z} \ddot{\psi}=F_{f}\left(\alpha_{f}\right) l_{f}-F_{r}\left(\alpha_{r}\right) l_{r}, \\
& m \ddot{y}=F_{f}\left(\alpha_{f}\right)+F_{r}\left(\alpha_{r}\right), \\
& \ddot{y}=v_{x}(\dot{\psi}+\dot{\beta}),
\end{aligned}
$$

where $m$ gives the mass and $I_{z}$ is the yaw-inertia of the vehicle. $F_{i}$ denotes the forces of the tires on the front $(f)$ and on the rear $(r)$ axle of the vehicle. The lateral position is given by $y$ and $\psi$ is the yaw-angle. The longitudinal velocity is denoted by $v_{x}$ and the side-slip of the vehicle is $\beta$. Finally, $l_{r}$ and $l_{f}$ are the distances between the center of gravity and the axis of the vehicle. The lateral forces of the tires can be computed as Eq. (8) (Rajamani, 2006):

$$
F_{i}=\alpha_{i} C_{i}, \quad i \in\{f, r\}
$$

where $C_{i}$ gives the cornering stiffness of the tires. Moreover, the side slip angles of the tires $\left(\alpha_{i}\right)$ can be determined as Eqs. (9), (10):

$$
\begin{aligned}
& \alpha_{f}=\delta-\beta-\frac{\psi l_{f}}{v_{x}}, \\
& \alpha_{r}=-\beta+\frac{\psi l_{f}}{v_{x}} .
\end{aligned}
$$

The trajectory planning is based on the bicycle model. Using the equations a state-space representation of the system can be determined, which can be formed as Eqs. (11), (12):

$$
\begin{aligned}
& \dot{x}=\boldsymbol{A} x+\boldsymbol{B} u, \\
& y=C^{T} x .
\end{aligned}
$$

Since the trajectory planning process requires a discrete time mode, it is transformed to the form described in Eq. (13)

$x(k+1)=\phi x(k)+\Gamma u(k)$,

where $\phi=e^{A T_{s}}$ and $\Gamma=\int_{k T_{s}}^{(k+1) T_{s}} e^{A\left((k+1) T_{s}-\tau\right)} \boldsymbol{B} \mathrm{d} \tau$. Moreover, $T_{s}$ gives the sampling time.

\subsection{Model Predictive Control (MPC) design}

In this subsection the Model Predictive Control design is presented. The states of the system can be computed for the $(k+1)^{\text {th }}$ time step as Eq. (14):

$x(k+1)=\phi x(k)+\Gamma u(k)$,

Using Eq. (14) the states are computable for the $(k+2)^{\text {th }}$ time step.

$$
\begin{aligned}
& x(k+2)=\phi x(k+1)+\Gamma u(k+1)=\phi(\phi x(k)+\Gamma u(k)) \\
& +\Gamma u(k+1)
\end{aligned}
$$

Using the initial states of the system and the input vector $(\boldsymbol{U}=[u(k+1), u(k+2) \ldots .]$.$) , the states can be computed$ for the arbitrary $(t+n)^{\text {th }}$ time step. Since, the reference lateral positions $(R)$ of the vehicle are already determined, the error can be computed as Eq. (16):

$\epsilon=y(\boldsymbol{U})-R$.

During the determination of the feasible trajectory, the following cost function must be minimized (Németh et al., 2019):

$J(\boldsymbol{U}, x(k), R)=\frac{1}{2} \sum_{i=k}^{k+n} \epsilon Q \epsilon+\boldsymbol{U}^{T} L \boldsymbol{U}$.

In Eq. (17) $Q$ and $L$ are weighting matrices. The optimal control vector can be determined by solving the quadratic optimization problem.

\section{Vertical control of the vehicle}

In this section the semi active suspension control is presented for the vehicle. In Section 3 the feasible trajectory is determined, with which the lateral accelerations can be calculated along the overtaking trajectory. This lateral acceleration vector is considered as a disturbance during the vertical control design. Furthermore, the main goal during the vertical control is to minimize the roll angle of the vehicle.

\subsection{The vertical dynamics of the vehicle}

In this section, the vertical dynamics of the vehicle are presented (Poussot-Vassal et al., 2008) in Fig. 5. Moreover, the control design process is detailed. 


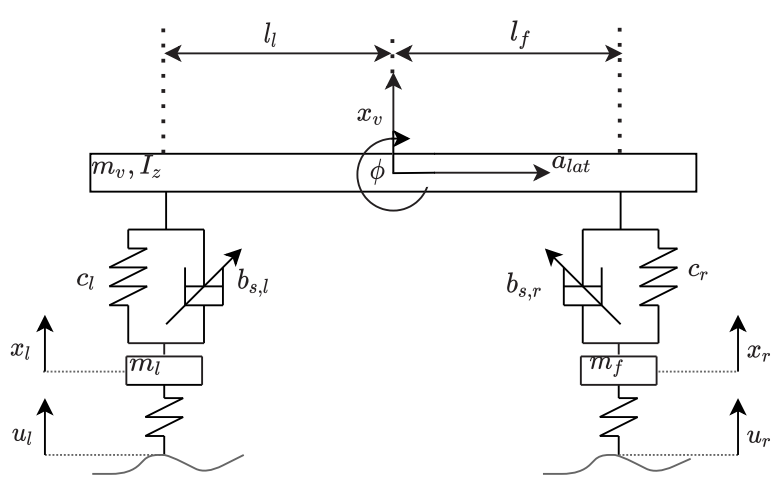

Fig. 5 Vertical dynamics of the vehicle

The acceleration and the roll acceleration of the sprung mass can be determined as Eqs. (18), (19):

$$
\begin{aligned}
& m_{v} \ddot{x}_{v}=c_{l}\left(x_{l}-x_{v}\right)+c_{r}\left(x_{r}-x_{v}\right)+b_{s, l}\left(\dot{x}_{l}-\dot{x}_{v}\right) \\
& +b_{s, r}\left(\dot{x}_{r}-\dot{x}_{v}\right)-\phi l_{l} c_{l}-\dot{\phi} l_{l} b_{s, l}+\phi l_{r} c_{r}+\dot{\phi} l_{r} b_{s, r}, \\
& I_{z} \ddot{\phi}=c_{l}\left(x_{l}-x_{v}\right) l_{l}+b_{s, l}\left(\dot{x}_{l}-\dot{x}_{v}\right) l_{l}-c_{r}\left(x_{r}-x_{v}\right) l_{r} \\
& -b_{s, r}\left(\dot{x}_{r}-\dot{x}_{v}\right) l_{r}-\phi l_{l}^{2} c_{l}-\phi l_{r}^{2} c_{r}-\dot{\phi} l_{r}^{2} b_{s, r}-\dot{\phi} l_{l}^{2} b_{s, l}-h_{c o g} m_{v} a_{l a t},
\end{aligned}
$$

where $\left(c_{r}, c_{l}\right)$ are the right and left suspension spring stiffnesses and $\left(l_{r}, l_{l}\right)$ gives the distances between the center of gravity and the suspensions. Moreover, $h_{c o g}$ denotes the height of the center of the gravity and $m_{v}$ is the sprung mass. Using Eqs. (18) and (19) the following state space representation can be formed in Eq. (20):

$\dot{\boldsymbol{x}}_{\mathrm{vertical}}=\boldsymbol{A} \boldsymbol{x}_{\text {vertical }}+\boldsymbol{B} u+\boldsymbol{B}_{a} a_{l a t}+\boldsymbol{B}_{r} u_{l, r}$,

where the state vector is the following: $\boldsymbol{x}_{\text {vertical }}$ $=\left[\dot{x}_{v}, \dot{x}_{l}, \dot{x}_{r}, \dot{\phi}, x_{v}, x_{l}, x_{r}, \phi\right]^{T}$. Since, the state space representation of the system would not be linear in the case, when the damping coefficients are chosen to be the control signals, a nominal $b_{s}$ is assumed, and the control signals are chosen to be the extra forces $\left(F_{l}, F_{r}\right)$ of the semi-active suspension. The state space representation must be discretized before the control design, which can be done similarly as it is described for the lateral case.

\subsection{Control design of the vertical dynamics}

An MPC is implemented for the vertical control of the vehicle. During the computation of the optimal control inputs, limitations can be made for the states of the system and also for the input signals. In this paper, the roll angle $(\phi)$ is minimized in order to fulfill comfort requirements. Using Eq. (17) the model predictive problem can be formulated as Eq. (21): $\min _{u(t+1), u(t+2) \ldots u(t+n)} J(\boldsymbol{U}, x(k), R)$

s.t. $l b \leq u_{i} \leq u b \forall i$,

where $(l b, u b)$ gives the lower and the upper bound of the input signals. Furthermore, the velocity of the unsprung mass is estimated using Eq. (18). This step is essential because the sign and magnitude of the force depend on the velocity difference between the sprung and unsprung masses. Using the estimated velocity value and the limitations of the actuator, the limitations $(l b, u b)$ can be computed. Fig. 6 depicts the control scheme of the vertical control system. Since the decision and trajectory planning algorithm provides the lateral accelerations, these values can be taken into account during the computation of the control inputs.

It can be said generally, that the value of the damping coefficients are set higher during the overtaking maneuver, and it should be decreased otherwise. In order to achieve this, the states are weighted during the optimization process, which is described in Eq. (17). The value of the weight for the roll is chosen as Eq. (22):

$Q_{\text {roll }, i}=0.1+a_{\text {lat }, i}^{2}$.

This means that during the overtaking maneuver, the damping coefficients are chosen to be higher values. Since the trajectory is designed beforehand, the weights can be computed for the given control horizon. Moreover, the weight of the input signal $(L)$ is set to 0.01 . In the following section a simulation example is presented, in which the controlled vehicle overtakes another car in front of it.

\section{Simulation of the proposed control system}

In this section a simulation example is presented, in which the controlled vehicle performs a double lane change maneuver. Moreover, several bumps are placed randomly along the given road segments in order to simulate bad road conditions. The goal is to fulfill comfort requirements during the overtaking maneuver using a lateral and vertical control of the vehicle. In Fig. 7 the roll angle is presented during the simulation.

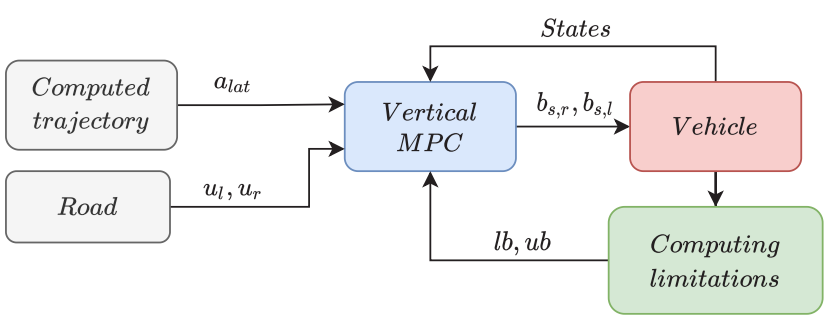

Fig. 6 Vertical control system 


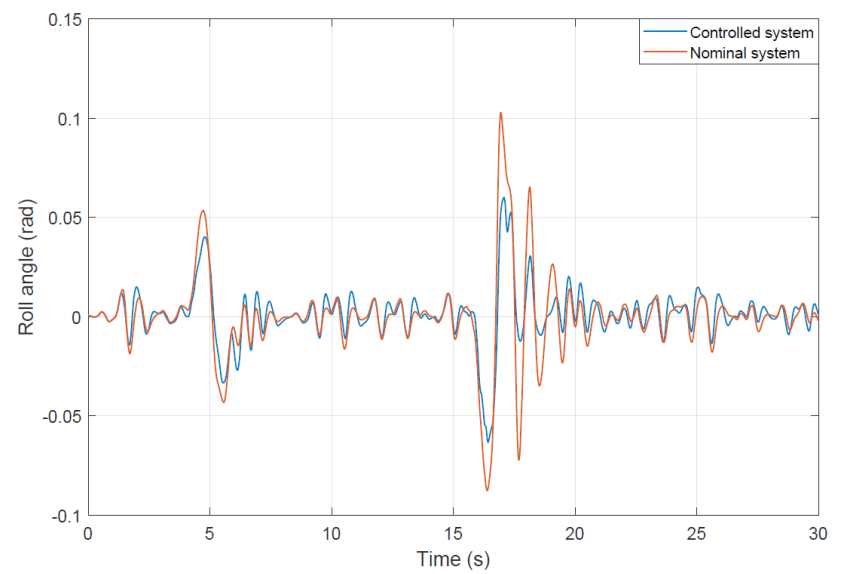

Fig. 7 Roll angle of the vehicle

The red line represents the case when the nominal suspension is used, which is provided by the CarMaker. Furthermore, the blue line shows the results of the controlled system. The first lane change maneuver is performed in the $4^{\text {th }} s$. The vehicle gets back to its original lane at the $15^{\text {th }} s$. It can be said that the roll angle during the lane change maneuvers decreased in the case, when the semi-active suspension is used. The computed control inputs are calculated for the half-vehicle model. This means that, in the figure the sum of the left and right coefficients can be seen, which is presented in Fig. 8.

Fig. 9 depicts the steering angle, which shows that the vehicle performed a double lane change maneuver.

Finally, Fig. 10 shows the lateral position of the controlled vehicle during the simulation.

It can be seen that at $\sim 350$ meters the vehicle starts to go to the wrong direction, this phenomenon can be explained by the fact that the trajectory planning algorithm also minimizes the lateral acceleration.

\section{Conclusion}

In this paper, a semi-active suspension design has been demonstrated for autonomous vehicles. During the control design, two main requirements have been guaranteed: safety and comfort. The safety requirements are achieved through a graph-based decision-making algorithm and a model predictive approach is applied for trajectory planning. During the trajectory planning, the maximum value of the curvature is minimized in order to fulfill the comfort requirements. Moreover, the vertical control is made by an MPC, which takes into account the previously computed lateral acceleration values. The whole simulation is performed in a vehicle dynamics simulation software, CarMaker. The results show that using the semi-active suspension improves the traveling comfort for the passengers.

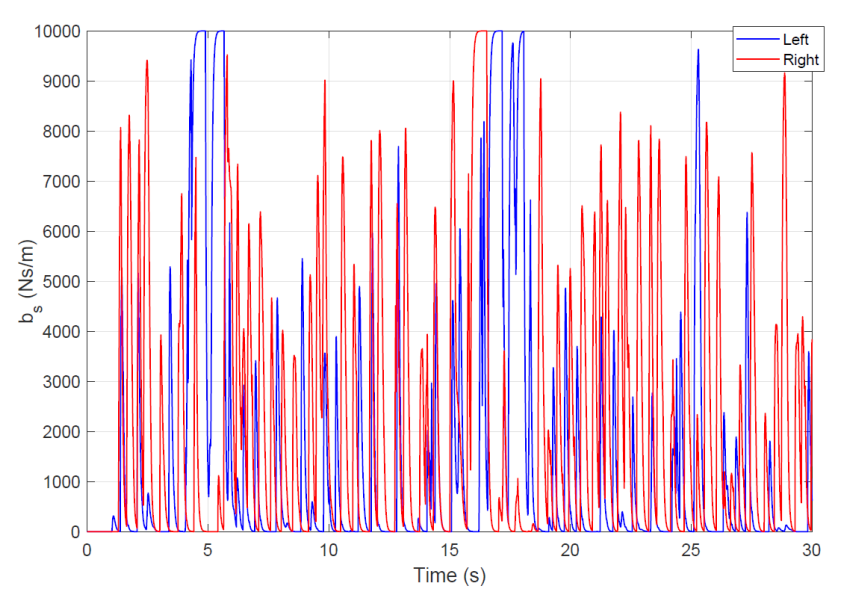

Fig. 8 The input signals of the system

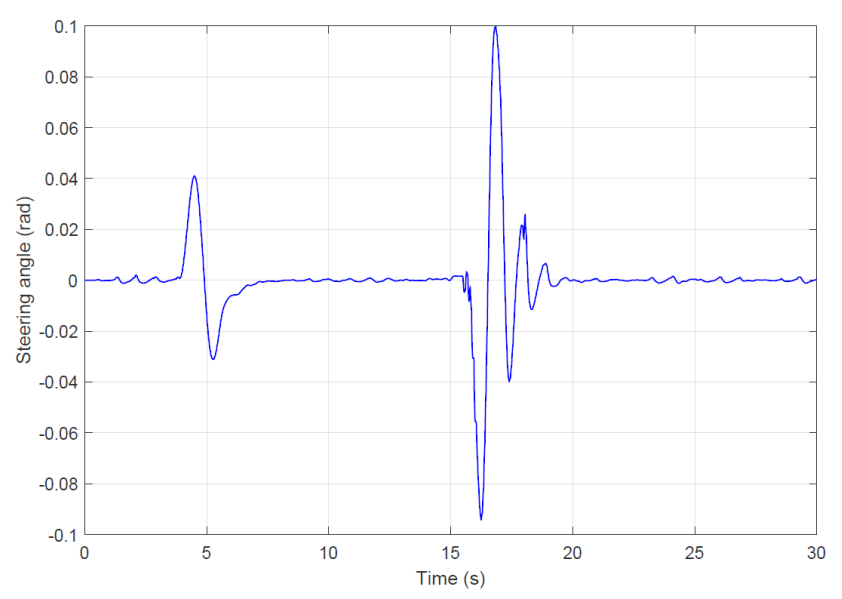

Fig. 9 Steering angle of the vehicle

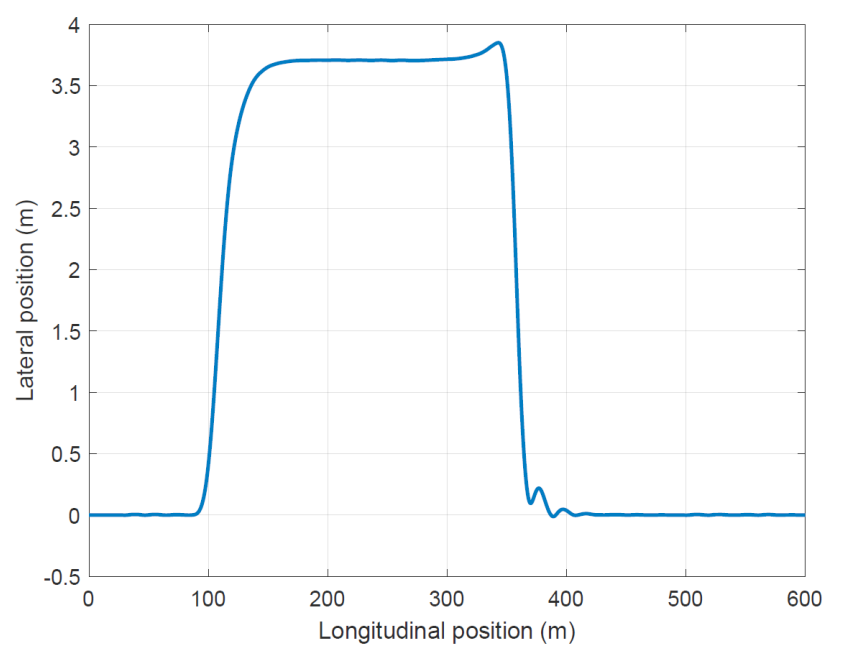

Fig. 10 Trajectory during the simulation example

\section{Acknowledgement}

The research was supported by the National Research, Development and Innovation Office through the project "Integration of velocity and suspension control to enhance automated driving comfort in road vehicles" (NKFIH 2018-2.1.13-TET-FR). 
The research was supported by the Ministry of Innovation and Technology NRDI Office within the framework of the Autonomous Systems National Laboratory Program.

The work of T. Hegedüs was supported by the ÚNKP20-3 New National Excellence Program of the Ministry for Innovation and Technology from the source of the National Research, Development and Innovation Fund.

\section{References}

Canale, M., Milanese, M., Novara, C., Ahmad, Z. (2005) "Semiactive suspension control using "fast" model predictive control", In: American Control Conference, Portland, OR, USA, pp. 274-281.

https://doi.org/10.1109/ACC.2005.1469945

Fang, Z., Shu, W., Du, D., Xiang, B., He, Q., He, K. (2011) "Semiactive Suspension of a Full-vehicle Model based on Double-loop Control", Procedia Engineering, 16, pp. 428-437. https://doi.org/10.1016/j.proeng.2011.08.1107

Fergani, S., Sename, O., Dugard, L. (2013) "A new LPV/H ${ }_{\infty}$ Global Chassis Control through load transfer distribution and vehicle stability monitoring", IFAC Proceedings Volumes, 46(2), pp. 809-804.

https://doi.org/10.3182/20130204-3-FR-2033.00188

Göhrle, C., Schindler, A., Wagner, A., Sawodny, O. (2013) "Model Predictive Control of semi-active and active suspension systems with available road preview", In: 2013 European Control Conference (ECC), Zurich, Switzerland, pp. 1499-1504. https://doi.org/10.23919/ECC.2013.6669185

Gui, L., Shi, W., Liu, W. (2012) "A semi-active suspension design for offroad vehicle base on Magneto-rheological technology", In: 2012 $9^{\text {th }}$ International Conference on Fuzzy Systems and Knowledge Discovery, Sichuan, China, pp. 2565-2568. https://doi.org/10.1109/FSKD.2012.6234078
The work of B. Németh was partially supported by the János Bolyai Research Scholarship of the Hungarian Academy of Sciences and the ÚNKP-20-5 New National Excellence Program of the Ministry for Innovation and Technology from the source of the National Research, Development and Innovation Fund.

Hegedűs, T., Németh, B., Gáspár, P. (2020) "Design of a Low-complexity Graph-Based Motion-Planning Algorithm for Autonomous Vehicles", Applied Sciences, 10(21), Article number: 7116. https://doi.org/10.3390/app10217716

Kanarachos, S. A., Spentzas, K. N. (2005) "Intelligent Semi-Active Suspension Control Applied to a Half-Car Model", In: 10 EAEC European Automotive Congress, Belgrade, Serbia, Vol. 3, pp. 1437-1448.

Németh, B., Hegedűs, T., Gáspár, P. (2019) "Model Predictive Control Design for Overtaking Maneuvers for Multi-Vehicle Scenarios", In: $201918^{\text {th }}$ European Control Conference (ECC), Naples, Italy, pp. 744-749. https://doi.org/10.23919/ECC.2019.8796013

Poussot-Vassal, C., Sename, O., Dugard, L., Gaspar, P., Szabo, Z., Bokor, J. (2008) "A new semi-active suspension control strategy through LPV technique", Control Engineering Practice, 16(12), pp. 1519-1534. https://doi.org/10.1016/j.conengprac.2008.05.002

Rajamani, R. (2005) "Vehicle Dynamics and Control", Springer, New York, NY, USA.

Unger, A., Schimmack, F., Lohmann, B., Schwarz, R. (2013) "Application of LQ-based semi-active suspension control in a vehicle", Control Engineering Practice, 21(12), pp. 1841-1850. https://doi.org/10.1016/j.conengprac.2013.06.006 